\title{
Comportamiento hidrodinámico de un reactor electroquímico para recuperar residuos en la fabricación de películas semiconductoras
}

\author{
Hudrodunamic Behavior of an Electrochemical Reactor for Waste \\ Recovery in the Semiconductor Films Manufacture
}

Luis Adrián Echeverri Holguín* Henry Reyes Pineda**

\section{Resumen}

En el laboratorio de optoelectrónica adscrito a la Universidad del Quindío se encuentra implementada la técnica Epitaxia en fase líquida, utilizada para elaborar películas semiconductoras de GaInAsSb, la cual genera, en promedio, un desecho de $1457 \mathrm{mg}$, constituido, en mayor proporción, por galio, indio y antimonio, que pueden ser recuperados por procesos electroquímicos. El propósito de este estudio fue reciclar y cristalizar estos metales puros a bajo costo, con cero productos de desechos metálicos en la fabricación de películas semiconductoras. Los estudios se llevaron a cabo en un reactor electroquímico de compartimentos separados, inyectando una solución trazadora de $\mathrm{NaCl}$ en el compartimento catódico, determinándose la conductividad a la entrada y a la salida del reactor, y describiendo con ello un modelo matemático del comportamiento hidrodinámico, mediante la

\section{Abstract}

In the optoelectronics laboratory affiliated to the University of Quindio it has been implemented the Liquid Phase Epitaxy Technique, used to elaborate semiconductor GalnAsSb films, which generates a waste with an average weight of 1457 $\mathrm{mg}$, a higher proportion is constituted by gallium, indium and antimony, which can be recovered by electrochemical processes. This study's purpose was to recycle and crystallize these pure metals at low cost, with zero metallic waste products in the semiconductor films manufacturing.

The studies were carried out in a compartments separated's electrochemical reactor, by injecting a $\mathrm{NaCl}$ tracer solution in the cathodic compartment, |determining the conductivity at the reactor inlet and outlet, thereby describing a hydrodynamic behavior's mathematical model, by distributing time residence (DTR) and thus applying it to these

\footnotetext{
* Universidad del Quindio (Colombia). laecheverrih@uqvirtual.edu.co

**Ph.D. Universidad del Quindio (Colombia). hreyes@uqvirtual.edu.co
} 
distribución de tiempos de residencia (DTR), y así aplicarlo para la recuperación de estos metales, operando el equipo a intensidad constante (modo galvanostático) y a potencial constante (modo potenciostático).

Palabras clave: Epitaxia, Semiconductor, Comportamiento hidrodinámico, Reactor electroquímico, Galvanostático, Potenciostático. metals recovery, operating the device at constant intensity (galvanostatic mode) and at constant potential (potentiostatic mode).

Keywords: $\quad$ Epitaxy, Semiconductor, Hydrodynamic Behavior, Electrochemical reactor, Galvanostatic, Potentiostatic. 


\section{INTRODUCCIÓN}

El semiconductor $\mathrm{Ga}_{1-\mathrm{x}} \mathrm{In}_{\mathrm{x}} \mathrm{As}_{\mathrm{y}} \mathrm{Sb}_{1-\mathrm{y}}$ tiene amplias aplicaciones en la industria de la optoelectrónica, para la fabricación de dispositivos con respuesta en el infrarrojo [1,2]; por ser un material cuaternario es posible variar independientemente y en un amplio rango los valores de la brecha de energía y del parámetro de red, lo que permite usar para su fabricación sustratos de diferentes materiales, presentando gran acople de red [3]. El rango de emisión del $\mathrm{Ga}_{1-\mathrm{x}} \mathrm{In}_{\mathrm{x}} \mathrm{As}_{\mathrm{y}} \mathrm{Sb}_{1-\mathrm{y}}$ fabricado sobre sustratos de $\mathrm{GaSb}$, con coincidencia de red, está entre 0.35 y $0.75 \mathrm{eV}[4,5]$, lo que lo hace interesante para aplicaciones en comunicaciones, ya que las fibras ópticas dopadas con flúor presentan muy baja atenuación en el infrarrojo cercano [6,7]; además, se emplea en la fabricación de detectores de contaminantes atmosféricos, diodos LED y láseres de infrarrojo, para aplicaciones en comunicaciones ópticas con bajas pérdidas de transmisión [8-11], y también para la construcción de celdas termofotovoltaicas [12].

Las películas semiconductoras de GaInAsSb pueden ser fabricadas mediante diversas técnicas, tales como epitaxia por haces moleculares (Molecular Beam Epitaxy-MBE), epitaxia en fase de vapor por gases metalorgánicos (Metalorganic Vapour Phase Epitaxy-OMVPE) y epitaxia en fase líquida (Liquid Phase Epitaxy-EPL) entre otras. La EPL es utilizada en la industria para fabricar dispositivos en masa, dado que ofrece la mejor relación entre calidad cristalina de la película fabricada y precio de fabricación [13]. El término de epitaxia se define como la deposición de una capa monocristalina sobre un sustrato monocristalino, de tal modo que la estructura cristalina de la película sea una continuación de la estructura del sustrato [14]. Esta técnica utiliza, para elaborar películas semiconductoras, soluciones líquidas sobresaturadas a altas temperaturas, a las que con un control en la composición estequiométrica de este cuaternario es posible fijar parámetros como la brecha de energía prohibida $E_{0}$ y el parámetro de red $a$, de acuerdo con el dispositivo que se quiere desarrollar; sin embargo, en la fabricación de estos semiconductores III-V se generan gran cantidad de residuos, que requieren ser recuperados usando procesos de reciclaje frecuentes, como la disolución térmica, la oxidación con oxígeno, la nitruración con atmósfera de amoniaco y la cloración con gas cloro [15]; no obstante, existen métodos electroquímicos que permiten la recuperación de galio, indio y antimonio en celdas convencionales por electrodeposición [16-19]. En general, las celdas electroquímicas han jugado papel importante en el avance tecnológico, con reactores como el de placas paralelas (tipo filtroprensa), que es el más comúnmente usado en aplicaciones a nivel de laboratorio y planta piloto. Por su parte, las celdas tipo filtro-prensa tienen aplicación principalmente en síntesis orgánica e inorgánica, celdas de combustible, baterías redox $\mathrm{y}$ en los procesos de tratamiento de efluentes [20]. En la literatura se encuentran trabajos con un gran número de reactores electroquímicos para diversos procesos [21-23]; sin embargo, no existe una aplicación directa para la recuperación de desechos de materiales semiconductores de GaInAsSb, fabricados por la técnica de epitaxia en fase líquida, menos aún mediante la utilización de reactores electroquímicos de compartimentos separados presentes en la aleación resultante de películas crecidas epitaxialmente [24].

En este estudio se propuso el diseño y desarrollo experimental de una celda de electrorrecuperación de galio, indio y antimonio basada en un reactor electroquímico de dos compartimentos, determinando el comportamiento hidrodinámico y su funcionamiento.

\section{Detalles Experimentales}

\section{A. Diseño de la celda}

El reactor electroquímico diseñado consta de dos compartimentos construidos en vidrio, con una capacidad de $250 \mathrm{~mL}$; los compartimientos fueron tubos de $60 \mathrm{~mm}$ de diámetro por 12 $\mathrm{cm}$ de altura, con flanches centrales, fondo redondo, desprendimiento inferior con olivas y desprendimiento superior izquierdo de diámetro interno $2 \mathrm{~cm}$ para la alimentación de la solución trazadora con abrazadera en policloruro de vinilo (PVC). Los compartimientos catódico y anódico 
están separados por membrana Nafion 117, para evitar mezcla de los productos de reacción en el cátodo y el ánodo, con migración selectiva del catión por depositar. La celda de electrodeposición se representa en la Fig. 1. Las ventajas de este reactor son la facilidad de funcionamiento y el bajo costo de los materiales utilizados.

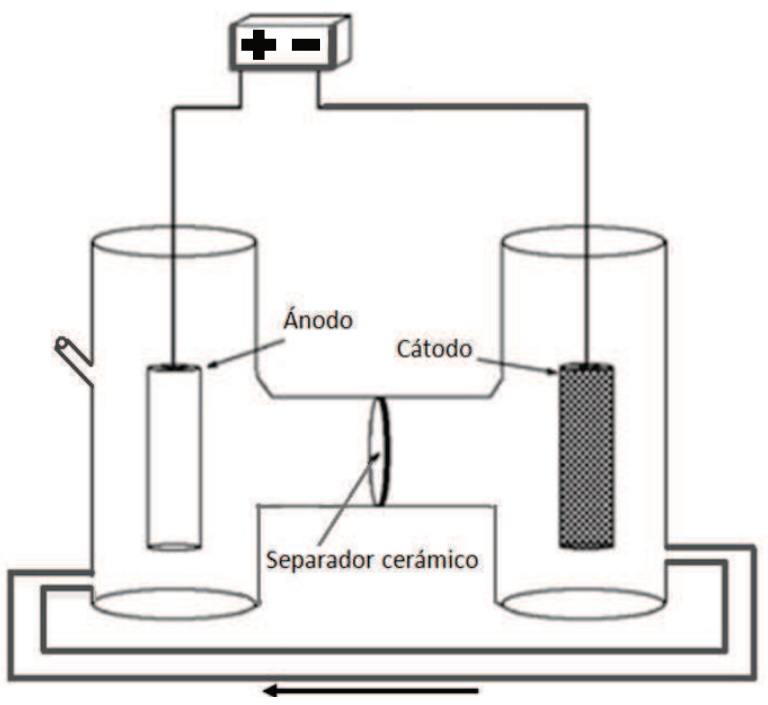

Fig. 1. Reactor de electrodeposición para la recuperación de $\mathrm{Ga}$, In y $\mathrm{Sb}$

\section{B. Electrodos}

El cátodo es una barra cilíndrica de grafito, de $8 \mathrm{~cm}$ de largo con $0,5 \mathrm{~cm}$ de diámetro, y el ánodo, de $\mathrm{Pb}$ Sn $(5 \%)$ tratado previamente con una solución de $\mathrm{H}_{2} \mathrm{SO}_{4} 1 \mathrm{M}$ durante dos días con el fin de obtener una película de óxido de plomo, fundamentalmente $\mathrm{PbO}_{2}$ sobre la superficie del electrodo, la cual es buena conductora de la corriente eléctrica y protege al electrodo de la corrosión. La geometría del ánodo es cúbica, con $8 \mathrm{~cm}$ de largo, $2 \mathrm{~cm}$ de ancho y $0,1 \mathrm{~cm}$ de espesor. Los electrodos se conectan a una fuente de alimentación para operar en modo potenciostático o en modo galvanostático. El compartimento anódico y el catódico se encuentran divididos por una membrana polimérica Nafion 117. Adicionalmente, los dos tanques están unidos a un sistema de recirculación por gravedad desde el compartimento catódico al anódico, para efectuar un máximo agotamiento de los cationes de interés en la solución electrolítica.

\section{Electrolitos}

Las soluciones electrolíticas utilizadas en este estudio fueron: en el compartimento de reducción, $100 \mathrm{~mL}$ de ácido sulfúrico 95-97\% P.A., suministrado por la casa científica Merk Colombia, y en el compartimento anódico, la aleación $\mathrm{Ga}$, In y $\mathrm{Sb}$ (residuo del proceso epitaxial) en $100 \mathrm{~mL}$ de ácido sulfúrico 95-97\% P.A.

Para determinar la distribución de tiempos de residencia, DTR, se utilizó una solución trazadora de cloruro de sodio ( $\mathrm{NaCl})$ 0,1 M [25-27], adicionada en el compartimento anódico en forma de impulsos mediante una jeringa, y midiéndose la evolución de la conductividad a la entrada y a la salida del reactor electroquímico de forma continua por medio de dos conductímetros. El fluido de trabajo es suministrado al reactor mediante una bomba con flujo de aire de $30 \mathrm{~mL}^{*} \mathrm{~min}^{-1}$.

\section{Resultados}

Para estudiar el comportamiento hidrodinámico del reactor electroquímico se determinó la distribución de tiempos de residencia (DTR), registrándose la variación de la conductividad en función del tiempo tanto a la entrada como a la salida del reactor.

En la Fig. 2 se representa la evolución de la conductividad a la entrada y a la salida del reactor respecto al tiempo para los datos experimentales obtenidos, considerándose un impulso normal. Tanto la curva a la entrada como a la salida tienen forma gaussiana y no son simétricas. La curva correspondiente a la salida es menos simétrica que la de la entrada, presentando una ligera cola indicativa de que dentro del reactor se produce un evidente grado de dispersión y de que existen zonas muertas o estancadas [28]. Adicionalmente, puede concluirse que no existen fenómenos de recirculación interna, al obtenerse un único pico a la salida. 


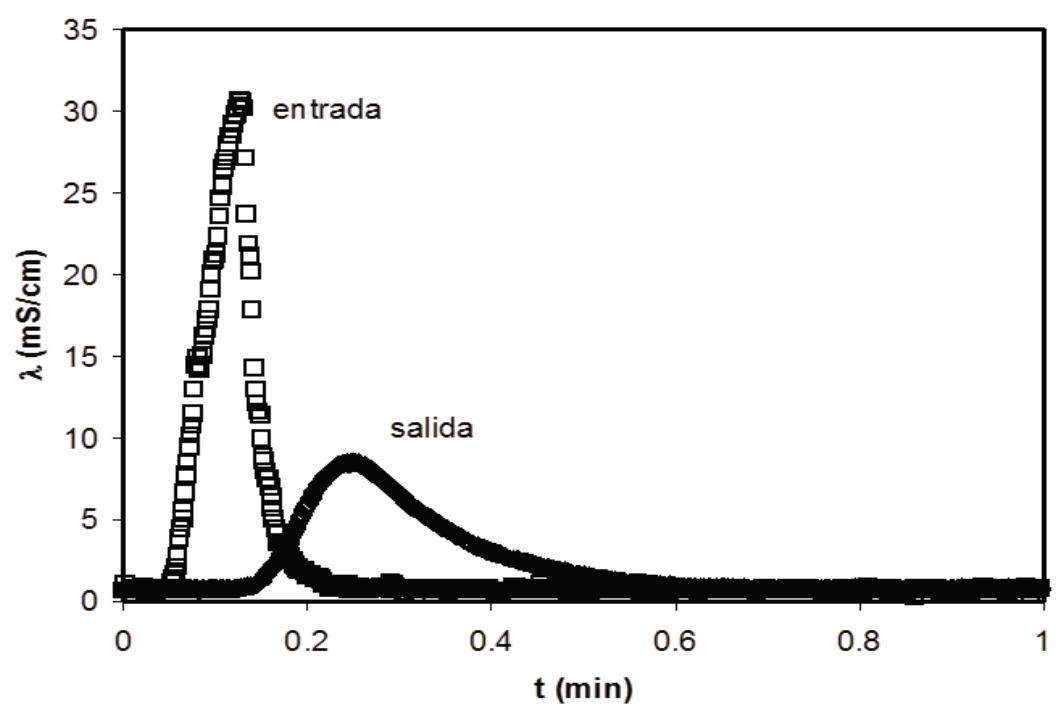

Fig. 2. Variación de la conductividad con respecto al tiempo a la entrada y a la salida del reactor electroquímico propuesto.

Para desarrollar un modelo del comportamiento hidrodinámico del reactor electroquímico $\mathrm{y}$ estimar sus parámetros característicos, se evaluó la conductividad normalizada tanto a la entrada como a la salida, calculada según la expresión (1).

$$
\lambda_{\text {norm }}=\frac{\lambda-\lambda_{0}}{\int_{0}^{t}\left(\lambda-\lambda_{0}\right) d t}
$$

Donde $\lambda$ es el valor de la conductividad a la entrada o a la salida en cualquier instante, y $\lambda_{0}$ es el valor de la conductividad inicial de la solución. Operando de esta forma, la curva de salida se corresponderá con la curva E, o curva de distribución de tiempos de residencia, de acuerdo con la expresión (2).

$$
\int_{0}^{t} E d t=1
$$

En la Fig. 3 se representa la evolución de la conductividad normalizada a la entrada y a la salida del reactor calculada a partir de los datos de la Fig. 2, aplicando la expresión (1). La forma de estas curvas es similar a las que se presenta en la Fig. 2, con la diferencia de que el área que hay por debajo de cada una de ellas equivale a la unidad.

En cualquiera de los dos casos de dispersión grande o pequeña para el comportamiento hidrodinámico del reactor, es necesario determinar el tiempo

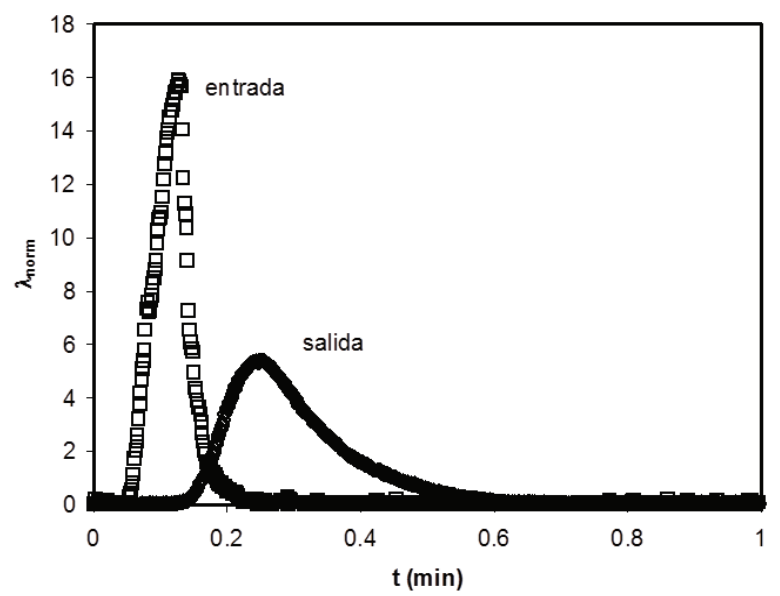

Fig. 3. Variación de la conductividad normalizada con el tiempo a la entrada y a la salida del reactor electroquímico propuesto

medio de residencia $t_{m}$, y la varianza $\sigma^{2}$, según las expresiones (3) y (4).

$$
\begin{aligned}
t_{m} & =\frac{\int_{0}^{\infty} t \cdot\left(\lambda-\lambda_{0}\right) d t}{\int_{0}^{\infty}\left(\lambda-\lambda_{0}\right) d t} \\
\sigma^{2}= & \frac{\int_{0}^{\infty} t^{2}\left(\lambda-\lambda_{0}\right) d t}{\int_{0}^{\infty}\left(\lambda-\lambda_{0}\right) d t}-t_{m}^{2}
\end{aligned}
$$


La ecuación general de diseño para este tipo de reactor electroquímico es una ecuación diferencial parcial de segundo orden que se expresa en forma adimensional de la siguiente manera [26, 29]:

$$
\frac{\partial C}{\partial \theta}=\left(\frac{D}{u L}\right) \frac{\partial^{2} C}{\partial Z^{2}}-\frac{\partial C}{\partial Z}
$$

Donde $\mathrm{Z}=(u t+z)^{*} L^{-1}$ y $\theta=\mathrm{t}^{*} \overline{\mathrm{t}}^{-1}=\mathrm{t} u^{*} L^{-1}$. El grupo adimensional $\left(D^{*} u L^{-1}\right)$, se denomina módulo de dispersión del reactor y es el parámetro que mide el grado de dispersión axial. Este cociente $D^{*} u L^{-}$

${ }^{1}$ es análogo al inverso del número de Péclet, mostrado en la expresión (6). $Z$ es la posición de la solución en cualquier instante t; $C$ es la concentración molar de la solución; $\theta$ es el tiempo de residencia en s; L es la longitud característica del reactor electroquímico en m; $D$ es el coeficiente de dispersión axial, y $u$ es la velocidad del fluido de trabajo.

$$
P e=\frac{u L}{D}
$$

Donde $u L$ representa la velocidad de transporte por convección.

La ecuación (5), en función del Número de Péclet y con unas condiciones de frontera dadas, puede expresarse de la siguiente forma (7):

$$
\frac{\partial C}{\partial \theta}=\frac{1}{P e} \frac{\partial^{2} C}{\partial Z^{2}}-\frac{\partial C}{\partial Z}
$$

Por lo tanto, para encontrar los parámetros característicos del comportamiento hidrodinámico del reactor electroquímico se resuelven dichas ecuaciones y se discretizan en la expresión (8), en donde la variable $x$ es la misma posición $Z$.

$$
t_{m} \frac{\partial C}{\partial t}=\frac{1}{P e} \frac{\partial^{2} C}{\partial x^{2}}-\frac{\partial C}{\partial x}
$$

Donde $\mathrm{t}_{\mathrm{m}}=L^{*} u^{-1}$ y $P e=L u^{*} D^{-1}$, y las condiciones iniciales y de frontera vienen dadas por (9), (10), (11) y (12).

$\mathrm{C}(0, x)=0$

$\mathrm{C}(\mathrm{t}, 0)=\delta(\mathrm{t})$

$$
\begin{gathered}
C\left(0^{-}, t\right)=C\left(0^{+}, t\right)-\frac{1}{P e}\left(\frac{\partial C}{\partial x}\right)_{x=n} \\
\left(\frac{\partial C}{\partial x}\right)_{x=L}=0
\end{gathered}
$$

Donde se asume que trabajar con concentraciones es equivalente a trabajar con conductividad normalizada, ya que en las condiciones de trabajo, la conductividad de la solución es directamente proporcional a la concentración.

La solución numérica de estas ecuaciones se resuelve por el método de diferencias finitas. Las derivadas parciales en el espacio y en el tiempo son discretizadas siguiendo el método de diferencias centrales, y la solución de estas ecuaciones permite calcular la variación de la conductividad con la posición y con el tiempo si se conoce $\mathrm{t}_{\mathrm{m}}$ y $D^{*} u L^{-}$ ${ }^{1}$. Los valores de la solución cuando $i=n$ son los que corresponden al valor de la conductividad a la salida del reactor electroquímico.

Para determinar los parámetros característicos de la DTR del reactor electroquímico, $\mathrm{t}_{\mathrm{m}}$ y $D^{*} u L^{-1}$, se realiza un ajuste de regresión no lineal mediante el método de Levenberg-Marquardt [30] entre los datos experimentales y los calculados mediante la solución de la ecuación diferencial (8) sometida a las condiciones de contorno dadas por las ecuaciones (9) a (12) y teniendo en cuenta los datos de la conductividad de entrada en cada caso. Para ello se operó de la siguiente forma: Se suponen unos valores iniciales de $\mathrm{t}_{\mathrm{m}}$ y $D^{*} u L^{-1}$, se resuelve la ecuación diferencial para obtener la conductividad normalizada a la salida, se comparan los valores obtenidos con los experimentales y se repite todo el proceso hasta conseguir el mejor ajuste de regresión no lineal siguiendo el método de Levenberg-Marquardt. Procediendo de esta forma se obtienen los siguientes valores de $\mathrm{t}_{\mathrm{m}} \mathrm{y} \mathrm{D} / u \mathrm{~L}$ :

$\mathrm{t}_{\mathrm{m}}=0.1426 \min \mathrm{y} \mathrm{D} / u \mathrm{~L}=0.1137$

Con estos valores y resolviendo la ecuación diferencial (8) se obtienen los resultados que se presentan en la Fig. 4, donde se compara la solución obtenida con los datos experimentales. Puede comprobarse que el modelo se ajusta perfectamente a los datos experimentales, por lo que puede concluirse que el comportamiento del reactor viene dado por las ecuaciones (9) a (12).

Finalmente, puede concluirse que la cola observada en la conductividad medida a la salida del reactor se debe a la forma de la entrada y no al hecho de que existan zonas muertas dentro del reactor. 


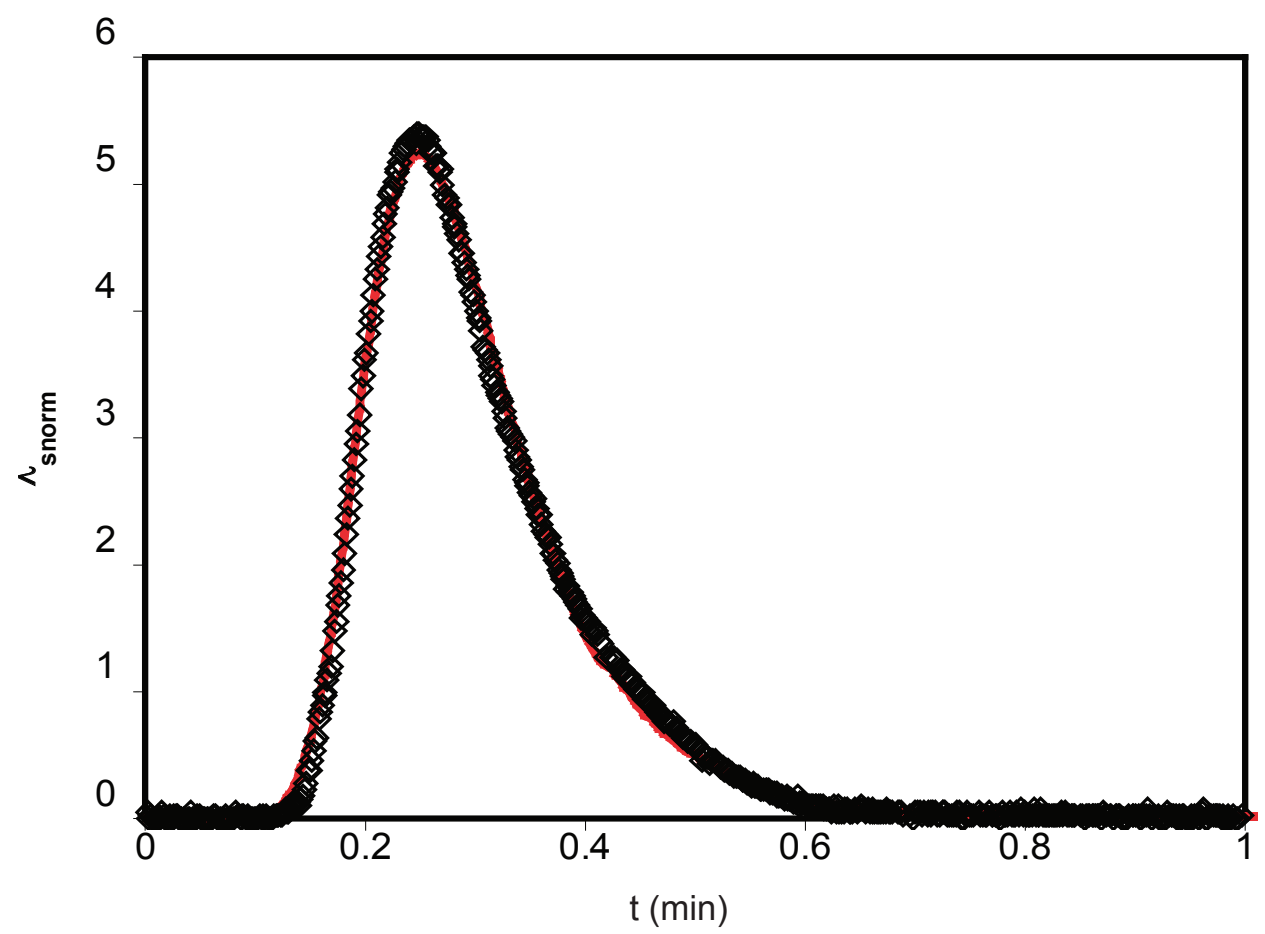

Fig. 4. Variación de la conductividad normalizada con el tiempo a la salida del reactor. Curva E.

$\diamond$ Datos experimentales — Solución numérica ecuación (8)

\section{Conclusiones}

El desarrollo del modelo matemático describe el comportamiento hidrodinámico de este tipo de reactor electroquímico de compartimentos separados, teniendo en cuenta su régimen de flujo, el cual puede ser escalado a nivel de planta piloto e industrial, revelando la importancia que tiene el estudio hidrodinámico como herramienta clave en su diseño.

Con base en estos resultados lo que sigue es continuar el estudio de la recuperación individual de $\mathrm{Ga}$, In y $\mathrm{Sb}$-elementos contenidos en la aleación remanente derivada del proceso de fabricación de películas semiconductoras por la técnica epitaxia en fase líquida del laboratorio de optoelectrónica afiliado a la Universidad del Quindío-,mediante la reducción a estado metálico directamente en un cátodo de grafito del reactor electroquímico a bajo consumo energético y tiempos de deposición cortos, factores convenientes para un posterior desarrollo industrial.

\section{REFERENCIAS}

[1] C. A. Wang, H. K. Choi, S. L. Ransom et al., "High-quantum-efficiency $0.5 \mathrm{eV} \mathrm{GaInAsSb/}$ GaSb thermophotovoltaic devices", Appl. Phys. Lett., Vol. 75, pp.1305-1308, July 1999.

[2] Yuan Tian, Tianming Zhou, Baolin Zhang et al., "Effect of material parameters on the quantum efficiency of GaInAsSb detectors", Solid State Electronics, Vol. 43, pp. 625-631, March 1999.

[3] M. J. Cherng, H. R. Jen, C. A. Larsen et al., "MOVPE growth of GaInAsSb", J. Cryst. Growth, Vol. 77, pp. 408-417, September 1986.

[4] O. Madelung und M. Schulz, Intrinsische Eigenschaften von Elemen der IV. Gruppe und III-V, II-VI und I-VII Verbindungen. Berlin, Deutschland, Springer Verlag, 1990.

[5] M. Levinshtein, S. Rumyantsev and M. Shurt, Editors, Handbook series on Semiconductor Parameters, Volume 2: Ternary and Quaternary $\mathrm{A}_{3} \mathrm{~B}_{5}$ Semiconductors, USA: 
Singapore World Scientific Publishing Co. Pte. Ltd.1999.

[6] E. Tournié, J. L. Lazzari, F. Pitard et al., "2.5 $\mu \mathrm{m}$ GaInAsSb lattice-matched to $\mathrm{GaSb}$ by liquid phase epitaxy", J. Appl. Phys., Vol. 68, pp. 5936-5939, July 1990.

[7] V. A. Mishurnyi, F. de Anda, A. Yu. Gorbatchev et al., "InGaAsSb growth from Sb-rich solutions", J. Cryst. Growth, Vol. 180, pp. 34-39, September 1997.

[8] K. Shim, H. Rabitz, and P. Dutta, "Band gap and lattice constant of $\mathrm{Ga}_{\mathrm{x}} \mathrm{In}_{1-\mathrm{x}} \mathrm{As}_{\mathrm{y}} \mathrm{Sb}_{1-\mathrm{y}}$ ", $J$. Appl. Phys., Vol. 88, pp. 7157-7161, Dec. 2000.

[9] K. Qiu, A. C. S. Hayden, M. G. Mauk et al., "Generation of electricity using InGaAsSb and GaSb TPV cells in combustion drivenradiant sources", Solar Energy Materials and Solar cells. Vol. 90, pp. 68-81, January 2006.

[10]K. Rößner, M. Hümmer, A. Benkert et al., "Long-wavelength GaInAsSb/ $\mathrm{AlGaAsSb}$ DFB lasers emitting near 2.6 $\mu \mathrm{m}$ ", Physica E: Low-dimensional Systems and Nanostructures, Vol. 30, pp. 159-163, December 2005.

[11] L. Cerutti, G. Boissier, P. Grech et al., "Growth and characterization of $\mathrm{GaInSb} / \mathrm{GaInAsSb}$ hole-well laser diodes emitting near $2.93 \mu \mathrm{m}$ ", J. Cryst. Growth, Volumes 301-302, pp. 967970, April 2007.

[12] Marín Muñoz, Fred H. Pollak, M.B. Zakia et al., "Temperature dependence of the energy and broadening parameter of the fundamental band gap of $\mathrm{GaSb}$ and $\mathrm{Ga}_{1-\mathrm{x}} \mathrm{In}_{\mathrm{x}} \mathrm{As}_{\mathrm{y}} \mathrm{Sb}_{1-\mathrm{y}} / \mathrm{GaSb}$ $(0.07<\sim \mathrm{x}<\sim 0.22,0.05<\sim \mathrm{y}<\sim 0.19)$ quaternary alloys using infrared photoreflectance", Phys. Rev. B, Vol. 62, pp. 16600-16604, December 2000.

[13] C.A. Wang, H. K. Choi, D.C. Oakley et al., "Recent progress in GaInAsSb thermophotovoltaics grown by organometallic vapor-phase epitaxy", J. Cryst. Growth, Vol. 195, pp. 346-355, December 1998.
[14] H. Ariza, "Fabricación y caracterización óptica de materiales semiconductores para aplicaciones en optoelectrónica". Rev. Acad. Colomb. Cienc. Vol. 27, pp. 357-368, Septiembre 2003.

[15] V. A. Fedorov, S. A. Kozlov, N. A. Potolokov et al., "Preparation of High-Purity Gallium from Semiconductor Fabrication Waste", Inorganic Materials, Vol. 42, pp. S70-S89, December 2006.

[16] Z. Zhao, Y. Yhag, Y. Xiao et al., "Recovery of gallium from Bayer liquor: A review", Hydrometallurgy, Vol. 125-126, pp. 115-124, August 2012.

[17]R.DorinandE.J.Frazer, “The electrodeposition of gallium from synthetic Bayer-process liquors". J. Appl. Electrochemistry, Vol. 18, pp. 134-141, January 1988.

[18] A.G. Muñoz, S.B. Saidman and J.B. Bessone, "Electrodeposition of $\mathrm{Zn}$ and In onto vitreous carbon", J. Appl. Electrochemistry, Vol. 29, pp. 1297-1304, November 1999.

[19] R. K. Iyer, and S. G. Deshpande, "Preparation of high-purity antimony by Electrodeposition", J. Appl. Electrochemistry, Vol. 17, pp. 936940, May 1987.

[20] F. C. Walsh, "Electrochemical technology for environmental treatment and clean energy conversion", Pure Appl. Chem., Vol. 73, pp. 1819-1837, April 2001.

[21] D. Pletcher and F. C. Walsh, Industrial Electrochemistry, Second Edition, London England, Chapman and Hall, 1990.

[22] F. C. Walsh, A First Course in Electrochemical Engineering, London, England, Romsey Electrochemical Consultancy, 1993.

[23] K. Jüttner, U. Galla and H. Schmieder, "Electrochemical approaches to environmental problems in the process industry", Electrochimica Acta, Vol. 45, pp. 2575-2594, January 2000. 
[24] P. Kiddee, R. Naidu and M. H. Wong, "Electronic waste management approaches: An overview. Review", Waste Management, Vol. 33, pp. 1237-1250, May 2013.

[25] L. R. P. Andrade Lima, "Liquid axial dispersion and holdup in column leaching", Minerals Engineering, Vol. 19, pp. 37-47, January 2006.

[26] H. Scott Fogler, Elements of Chemical Reaction Engineering, Third Edition, pp. 895, New Jersey USA., Prentice Hall Inc. (now known as Pearson Education, Inc.), 1999.

[27] V. Pérez-Herranz, J.L. Guiñón, J. GarcíaAntón et al., "Regeneration of etching solutions using electrochemical reactors provided", 16th International Congress of
Chemical and Process Engineering CHISA (Prague, Czech Republic), pp. 1-10, Process Engineering Publisher, 22nd-26th August 2004.

[28] L. Szpyrkowicz, R. Cherbanski, and G. Kelsall, "Hydrodynamic Effects on the Performance of an Electrochemical Reactor for Destruction of Disperse Dyes". Ind. Eng. Chem. Res., Vol. 44, pp. 2058-2068, February 2005.

[29] O. Levenspiel, Chemical reaction engineering, Third Edition, pp. 668, New York USA., Ed. John Wiley and Sons.1999.

[30] E. Tyrtyshnikov, A brief introduction to numerical analysis, pp. 216, Moscow Russia, Ed. Birkhäuser Boston, July 1997. 